\title{
Mechanical properties of particles from the surface of asteroid 25143 Itokawa
}

\author{
Safoura Tanbakouei ${ }^{1,2}$, Josep M. Trigo-Rodríguez ${ }^{1,2}$, Jordi Sort ${ }^{3,4}$, Patrick Michel ${ }^{5}$, Jürgen Blum ${ }^{6}$, \\ Tomoki Nakamura ${ }^{7}$, and Iwan Williams ${ }^{8}$ \\ ${ }^{1}$ Institute of Space Sciences (ICE-CSIC), Campus UAB, C/Can Magrans s/n, 08193 Bellaterra (Barcelona), Catalonia, Spain \\ e-mail: tanbakouei@ice.csic.es \\ ${ }^{2}$ Institut d'Estudis Espacials de Catalunya (IEEC), C/Gran Capità, 2-4, Ed. Nexus, desp. 201, 08034 Barcelona, Catalonia, Spain \\ ${ }^{3}$ Departament de Fisica, Universitat Autònoma de Barcelona, 08193 Bellaterra, Catalonia, Spain \\ ${ }^{4}$ Institució Catalana de Recerca i Estudis Avançats, Passeig Lluís Companys 23, 08010 Barcelona, Catalonia, Spain \\ ${ }^{5}$ Université Côte d'Azur, Observatoire de la Côte d'Azur, CNRS, Lagrange Laboratory, France \\ ${ }^{6}$ Institut für Geophysik und extraterrestrische Physik, Technische Universität Braunschweig, Mendelssohnstr. 3, \\ 38106 Braunschweig, Germany \\ ${ }^{7}$ Department of Earth Science, Graduate School of Science, Tohoku University, Japan \\ ${ }^{8}$ School of Physics and Astronomy, Queen Mary, University of London, Mile End Rd. London E1 4NS, UK
}

Received 28 February 2019 / Accepted 10 July 2019

\begin{abstract}
Aims. Asteroids have been exposed to impacts since their formation, and as a consequence their surfaces are covered by small particles, pebbles, and boulders. The Japanese JAXA/ISAS Hayabusa mission collected micron-sized particles from the regolith of asteroid 25143 Itokawa. The study in terrestrial laboratories of these particles provides a scientific opportunity as their physical properties can be compared with those characteristic of chondritic meteorites that are often considered proxies of the building materials of potentially hazardous asteroids (PHAs).

Methods. Here we present the results from a study of the mechanical properties of three of these particles using a precise technique called nanoindentation. The derived results are compared with those obtained via a methodology similar to that used for the Chelyabinsk meteorite.

Results. The reduced Young's modulus values obtained for the Itokawa samples are higher than those measured for the Chelyabinsk chondrite, so these specific particles of asteroid regolith are more compacted than the minerals forming the particular LL chondrite associated with PHAs. This might be a natural consequence of particles surviving long exposure times on the surface of a (near-Earth asteroid) NEA. The Double Asteroid Redirection Test (DART) mission plans to excavate a crater in the surface of the (65803) Didymos satellite. Our results suggest that excavating a crater with a kinetic impactor in an area of significant fine-grained regolith will increase the momentum transfer. As this will facilitate the release of particles carrying target mass in the opposite direction to the movement of the projectile, there is no need to grind up the target during the mechanical excavation phase.
\end{abstract}

Key words. minor planets, asteroids: general - methods: analytical - meteorites, meteors, meteoroids

\section{Introduction}

The JAXA/ISAS Hayabusa spacecraft had a rendez-vous with asteroid 25143 Itokawa, and collected regolith samples from its surface after landing on the asteroid in November 2005. The collector sample-return capsule returned to Earth in June 2010 (Yano et al. 2006). The remote study of Itokawa reflectance properties revealed that the asteroid spectrum was consistent with that of an S-type asteroid and its bulk mineralogy characteristic of the LL group of ordinary chondrites (Abe et al. 2006). The surface of Itokawa consists of large stones and rocks, confirmed by the analysis of the return sample (Nakamura et al. 2011), which are not uniformly distributed, and a regolith essentially made of pounded stones and gravel-sized particles (Saito et al. 2006). A variety of boulders ranging from meters to hundreds of meters in diameter were also identified by the Hayabusa spacecraft (Saito et al. 2006). The re-arrangement of rocks and boulders on the surface of this rubble pile asteroid and the observed displacement of the regolith can be explained by collisions and related processes (Saito et al. 2006; Matsumoto et al. 2016).

25143 Itokawa belongs to the S-class, one of the most abundant asteroid spectral types, and space missions like Hayabusa can help provide an answer to how similar their properties are in comparison with ordinary chondrites (Burbine 2002). To decipher the physical properties of asteroidal regolith is of great relevance for space exploration. Different techniques have been developed to infer the properties of the regolith from remote observations (Hiroi et al. 1994), but in situ measurements are still scarce. On the other hand, it is well known that meteorite collections are strongly biased toward high tensile strength rocks, so we lack sufficent regolith materials for the direct study in terrestrial laboratories (Chapman 2002).

To date, most of our current understanding of regolith comes from the study of the lunar regolith sampled by the Apollo and other Moon missions (Papike et al. 1982). There are good reasons to study the physical properties of asteroid regolith in order to know the possible differences between lunar and asteroidal 
regolith. Obviously, the first sample-returned regolith from an asteroid by Hayabusa spacecraft has focused the attention of the scientific community. Remote observations, laboratory studies, and space missions have revealed that regolith covers the surface of many asteroids, and might be produced by very different physical processes including the comminution of the surface rock-forming minerals, including impact gardening and thermal fatigue (Delbo et al. 2014). For these reasons, sample return missions provide access to unique materials from which valuable data on the physical properties of regolith particles can be extracted. In addition, the properties of the surface materials from well-known asteroids are inferred from these studies. This work constitutes one of the first studies on the mechanical properties of asteroid regolith materials that might be of interest to future sample-return missions.

In this paper we gain insight on the mechanical properties of asteroid Itokawa's regolith by studying three micron-sized particles collected by the Hayabusa JAXA mission. The data obtained are compared with the materials forming chondritic meteorites. One of the questions to be addressed is whether collisional gardening can change the main mechanical properties of the materials forming the surface of a rubble-pile asteroid like Itokawa (Beitz et al. 2016).

\section{Instrumental procedure}

The mechanical properties of the specimens, which were embedded in resin and polished using diamond paste, were evaluated by nanoindentation (UMIS Equipment from Fischer-Cripps Laboratories). During nanoindentation a controlled load is applied to some parts of a sample through the use of a diamond indenter. The indenter pushes the surface while the load is increased to a specified maximum. After loading, the force is progressively brought back to zero and the sample surface retracts because of elasticity. The penetration depth is acquired during the whole load-unload cycle. According to the extracted loaddepth curves, useful information concerning the deformation mechanisms (elastic and plastic) and the elastic recovery are obtained.

The mechanical properties such as hardness and the reduced Young's modulus, were evaluated by the method developed by Oliver \& Pharr (1992). We chose applied forces up to $10 \mathrm{mN}$ in order to keep the maximum penetration depth below one-tenth of the overall thickness of the sample and to minimize the eventual influence of the resin on the obtained results Fischer-Cripps (2004). We applied some necessary corrections for the contact area and compliance of the instrument. We also kept the thermal drift below $0.05 \mathrm{~nm} \mathrm{~s}^{-1}$. The contact stiffness is defined as

$S=\frac{\mathrm{d} P}{\mathrm{~d} h}$,

where $P$ denotes the applied load and $h$ the penetration depth during nanoindentation. The maximum load applied is denoted $P_{\max }$. The hardness is calculated as

$H=\frac{P_{\max }}{A}$,

where $A$ is the projected contact area on the horizontal plane. The elastic modulus can be evaluated based on its relationship with the contact area $A$ and the contact stiffness:

$S=\beta \frac{2}{\sqrt{\pi}} E_{\mathrm{r}} \sqrt{A}$.
Table 1. Main composition of the studied regolith samples.

\begin{tabular}{lcl}
\hline \hline Sample & Size $(\mu \mathrm{m})$ & Main mineral phases \\
\hline$\# 14$ & $131.2 \pm 0.1$ & Olivine, low-Ca pyroxene, plagioclase \\
$\# 23$ & $149.4 \pm 0.1$ & Olivine, troilite \\
$\# 47$ & $108.0 \pm 0.1$ & Olivine, low-Ca and high-Ca pyroxene \\
\hline
\end{tabular}

Here $\beta$ is a constant that depends on the geometry of the indenter ( $\beta=1.034$ for a Berkovich indenter; Fischer-Cripps 2004) and $E_{\mathrm{r}}$ is the reduced Young's modulus defined as follows:

$\frac{1}{E_{\mathrm{r}}}=\frac{1-v^{2}}{E}+\frac{1-v_{\mathrm{i}}^{2}}{E_{\mathrm{i}}}$.

The reduced modulus takes into account the elastic displacements that occur in both the specimen, with Young's modulus $E$ and Poisson's ratio $v$, and in the diamond indenter, with elastic constants $E_{\mathrm{i}}$ and $v_{\mathrm{i}}$. The data for diamond were $E_{\mathrm{i}}=1140 \mathrm{GPa}$ and $v_{\mathrm{i}}=0.07$.

We also evaluated the elastic recovery as the ratio of the elastic indentation energy $U_{\mathrm{el}}$ (calculated as the area enclosed between the unloading indentation curve and the $x$-axis) to the total indentation energy $U_{\mathrm{tot}}=U_{\mathrm{el}}+U_{\mathrm{pl}}$ (i.e., the area between the loading indentation section and the $x$-axis).

Three Itokawa particles provided by JAXA embedded in epoxy resin and polished to mirror-like appearance, with numbers RA-QD02-0014, RA-QD02-0023, and RA-QD02-0047 (hereafter S14, S23, and S47, respectively) were investigated (see Table 1). The polished upper sides of the three particles are shown in Fig. 1. The samples were analyzed via optical microscopy and scanning electron microscopy (Quanta 650 FEG equipped with EDX Inca 250 SSD XMax20 detector).

Indentations were applied on the different mineral phases contained in the three particles with applied forces of 5 and $10 \mathrm{mN}$, respectively, in order to extract the nanomechanical properties of the samples. To calculate the gross mechanical properties of the samples the results of the individual indentations applied on the mineral phases were averaged. The results of multiple indentations provided an estimation of the average mechanical properties of different regions, including the uncertainties based on natural deviations in the rock-forming materials.

\section{Results and discussion}

It is of great interest to understand the mechanical properties of the studied regolith grains because 25143 Itokawa can be considered a clear example of a rubble-pile asteroid (Saito et al. 2006). Cratering in asteroids has been studied in other asteroids as well, and the nature and properties of asteroidal regolith seem to be particularly interesting for future exploration missions to minor bodies (Chapman 2004). At the micrometer scale of our samples, Itokawa's regolith can be considered a good example of the materials forming the surface of an ordinary chondrite (OC) asteroid with recognizable lithologies LL4-6. In addition, Itokawa's surface consists of many chondritic fragments that are thermally metamorphosed or fragmented, having experienced the implantation of other nanoscale particles as a consequence of space weathering (Nakamura et al. 2011 and Noguchi et al. 2011). It is also possible that some regolith grains on the surface of asteroids were created by thermal fatigue (Matsumoto et al. 2016). We are going to concentrate on the main mechanical properties inferred for the studied Itokawa regolith grains and the main implications of these results. 

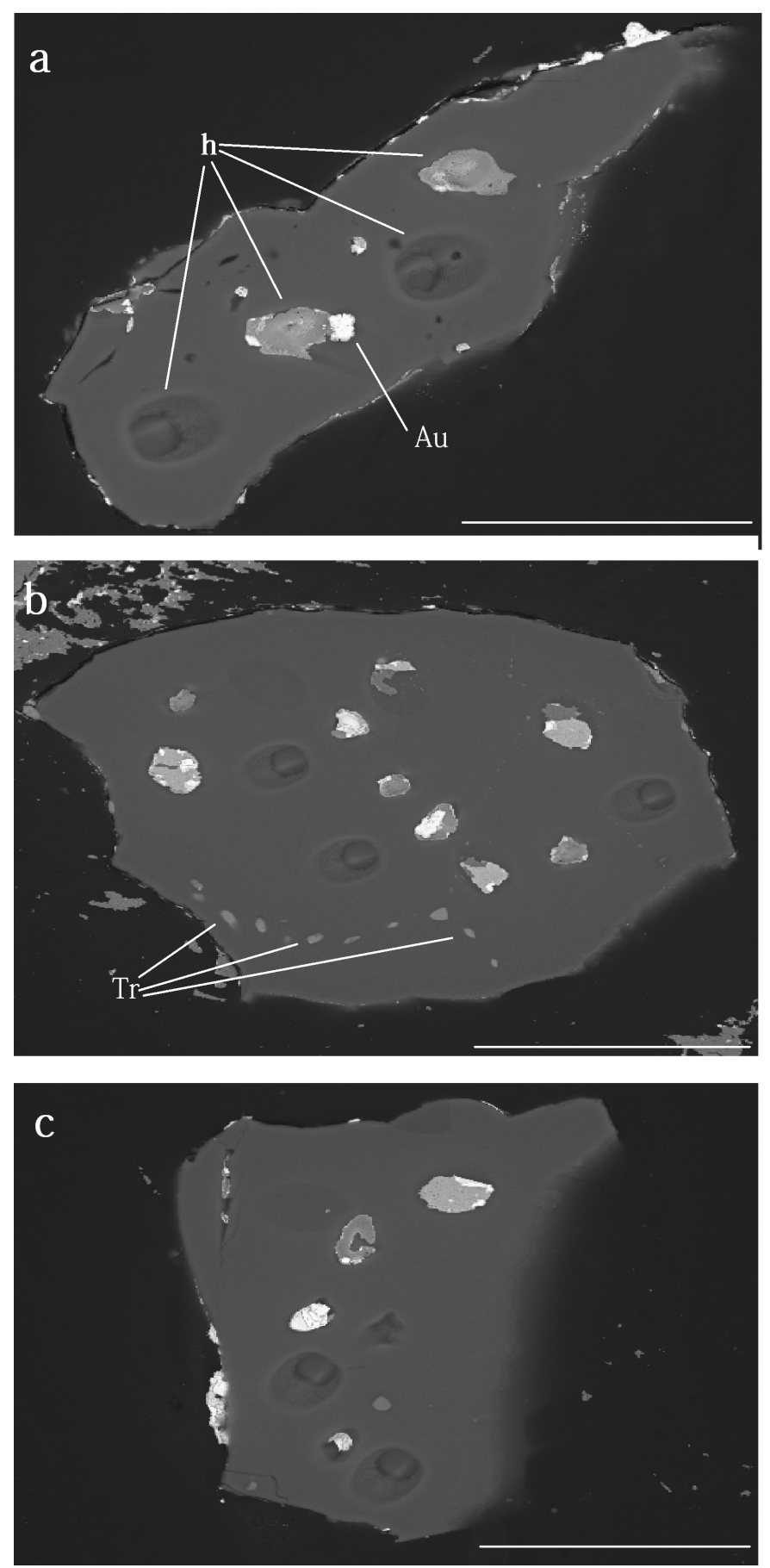

Fig. 1. BSE images of the three Itokawa grains. (a) RA-QD02-0014, (b) RA-QD02-0023, and (c) RA-QD02-0047. The white scale bars are 50,70 , and $60 \mu \mathrm{m}$ respectively. Labeled in panel $a$ are several holes (h) and $\mathrm{Au}$ patches from previous analyses. A chain of troilite (tr) grains is also labeled in panel $b$.

Mechanical properties. Representative nanoindentation curves for the three studied particles are shown in Fig. 2, from which the mean mechanical properties resulting from the analyzed region can be extracted (see Table 2).

As the values of hardness are not remarkably different at 5 and $10 \mathrm{mN}$, it seems that within this indentation force range, the hardness is not affected by the maximum applied load (Nix \& Gao 1998). This demonstrates that with our chosen loads, hardening phenomena, which could result from the indentation size effect, are avoided.
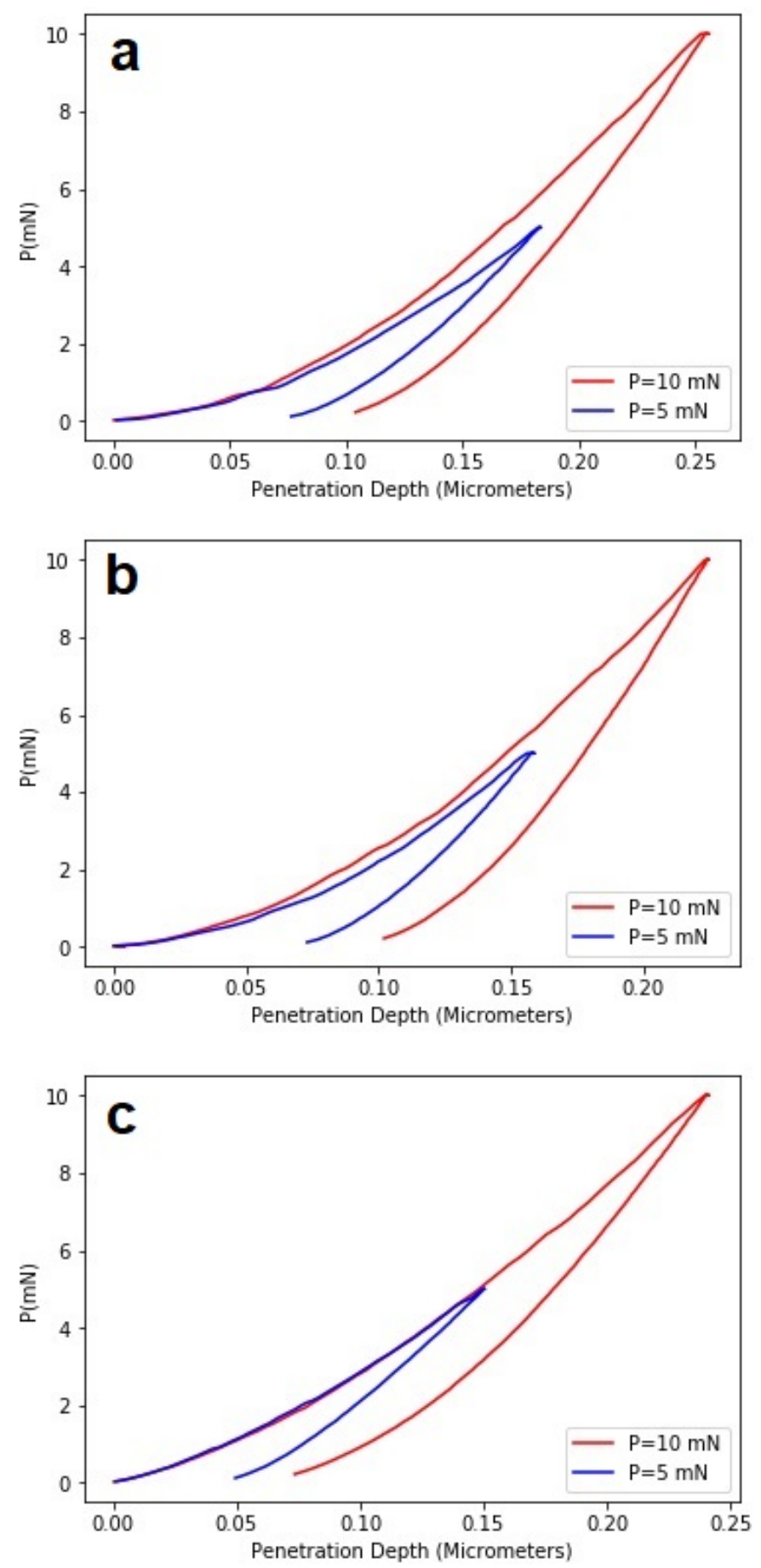

Fig. 2. Indentation curves obtained for Itokawa samples. (a) Indentation curves obtained for $\mathrm{S} 14$ sample. (b) Indentation curves obtained for S23 sample. (c) Indentation curves obtained for S47 sample.

We compare our results with the mechanical properties of the Chelyabinsk LL chondrite specimens obtained with a similar methodology during the nanoindentation experiments (UMIS Nanoindentor from Fischer-Cripps Laboratories; Moyano-Cambero et al. 2017). The particles in both cases were embedded in a resin and subsequently polished to mirror-like appearance using analogous polishing procedures. The size of the particles (hundreds of micrometers) is much larger than the indentation imprint size (on the order of $12 \mu \mathrm{m}$, depending on the applied indentation load). Therefore, no effect from the surrounding resin on the evaluated mechanical properties is 
Table 2. Average mechanical properties of Itokawa regolith silicates in particles of given $\mathrm{S \#}$.

\begin{tabular}{lcccccc}
\hline \hline $\begin{array}{c}\text { Applied } \\
\text { force } \\
(\mathrm{mN})\end{array}$ & $E_{\mathrm{r}}$ & $H$ & $S$ & $U_{\mathrm{el}} / U_{\text {tot }}$ & $U_{\mathrm{pl}} / U_{\text {tot }}$ \\
\hline$\# 14$ & 5 & $83.01 \pm 0.12$ & $8.01 \pm 0.01$ & $77.01 \pm 0.11$ & $0.64 \pm 0.01$ & $0.36 \pm 0.01$ \\
& 10 & $86.00 \pm 0.04$ & $9.00 \pm 0.014$ & $105.00 \pm 0.06$ & $0.66 \pm 0.01$ & $0.34 \pm 0.01$ \\
\hline$\# 23$ & 5 & $111.01 \pm 0.22$ & $10.01 \pm 0.02$ & $92.00 \pm 0.20$ & $0.73 \pm 0.07$ & $0.27 \pm 0.07$ \\
& 10 & $101.00 \pm 0.05$ & $10.00 \pm 0.01$ & $117.0 \pm 0.5$ & $0.66 \pm 0.05$ & $0.34 \pm 0.05$ \\
\hline$\# 47$ & 5 & $86.01 \pm 0.13$ & $13.01 \pm 0.03$ & $62.01 \pm 0.03$ & $0.87 \pm 0.01$ & $0.13 \pm 0.01$ \\
& 10 & $82.00 \pm 0.14$ & $11.00 \pm 0.02$ & $91.01 \pm 0.07$ & $0.68 \pm 0.01$ & $0.32 \pm 0.01$ \\
\hline
\end{tabular}

Notes. Reduced Young's modulus $\left(E_{\mathrm{r}}\right)$, hardness $(H)$, constant stiffness $(S)$, elastic recovery $\left(U_{\mathrm{el}} / U_{\mathrm{tot}}\right)$, and plasticity index $\left(U_{\mathrm{pl}} / U_{\mathrm{tot}}\right)$ were calculated by averaging the results from two lines of indentations from the maximum applied force of $10 \mathrm{mN}$.

expected. The mechanical properties were measured by nanoindentation applied on the different mineral parts of this OC with a maximum applied force of $20 \mathrm{mN}$. For the Chelyabinsk meteorite the reduced Young's modulus was calculated between 69 and $78 \mathrm{GPa}$ (Moyano-Cambero et al. 2017), which is lower than the values obtained for Itokawa's samples of 82-111 GPa (Table 2). Furthermore, the Young's modulus measured previously by Yomogida \& Matsui (1983) for OCs, between 10 and $140 \mathrm{GPa}$, is quite consistent with the values inferred for Itokawa's regolith grains. Our measurements show lower hardness values than in the olivine forming the Chelyabinsk meteorite, 10.2 and 13.6 GPa, respectively (Moyano-Cambero et al. 2017). Assuming that the composition of the two investigated objects is similar (similar minerals and with similar percentages), the lower Young's modulus observed in Chelyabinsk suggests that it contains higher porosity levels, since porosity has a strong detrimental effect on the elastic properties of materials (Pellicer et al. 2012). Different models exist in the literature to correlate the Young's modulus with porosity, both for metallic and ceramic materials (Tolu et al. 2013). In a first approximation, it has been shown that (Pellicer et al. 2012)

$\frac{E_{\text {porous }}}{E_{\text {bulk }}}=\left(\frac{\rho_{\text {porous }}}{\rho_{\text {bulk }}}\right)^{2}$,

where the right-hand term is the relative density and the coefficient $n$ depends on the material (in other words, $n=2$ in samples with an open-cell porosity (Tolu et al. 2013). This relative density can be related to the material porosity volume fraction $P_{\mathrm{o}}$ :

$\frac{\rho_{\text {porous }}}{\rho_{\text {bulk }}}=1-P_{\mathrm{o}}$.

It is well known that even a low degree of porosity can result in a drastic reduction in the Young's modulus. This effect is considered in the approach by Ramakrishnan and Arunachalam, which includes the intensification effects of pressure at the surface of spherical pores due to interactions between the pores and the surrounding bulk solid material (Ramakrishnan \& Arunachalam 1993). For a given porosity fraction the elastic modulus of the porous material ( $\left.E_{\text {porous }}\right)$ can be then related to the elastic modulus of the fully dense material $\left(E_{\text {bulk }}\right)$ following

$\frac{E_{\text {porous }}}{E_{\text {bulk }}}=\frac{\left(1-P_{\mathrm{o}}\right)^{2}}{1+2 P_{\text {or }}-3 v P_{\text {or }}}$,

where $v$ is the Poisson ratio of the bulk nonporous material.
Table 3. Estimated mass of the three Itokawa particles assuming silicate density of $2.2 \times 10^{-12}\left(\mathrm{~g} \mu \mathrm{m}^{-3}\right)$ taking their porosity into account.

\begin{tabular}{lcccc}
\hline \hline Sample & $\begin{array}{c}\text { Volume } \\
\left(\mu \mathrm{m}^{3}\right)\end{array}$ & $\begin{array}{c}\text { Estimated } \\
\text { mass }(\mathrm{g})\end{array}$ & $\begin{array}{c}\text { Porosity } \\
(\%)\end{array}$ & $\begin{array}{c}\text { Estimated mass } \\
\text { with porosity }(\mathrm{g})\end{array}$ \\
\hline$\# 14$ & 125921 & $2.76 \times 10^{-7}$ & 0.7 & $2.75 \times 10^{-7}$ \\
$\# 23$ & 781081 & $1.72 \times 10^{-6}$ & 0.0 & $1.71 \times 10^{-6}$ \\
$\# 47$ & 148570 & $3.26 \times 10^{-7}$ & 0.0 & $3.26 \times 10^{-7}$ \\
\hline
\end{tabular}

Notes. The porosity values are from Tsuchiyama et al. (2014).

In the Chelyabinsk compositions, olivine ( $\sim \mathrm{Fa} 28)$ is two to four times more abundant than pyroxene ( Fs23) (Galimov et al. 2013). However, the two minerals are homogeneous in composition (Galimov et al. 2013; Kohout et al. 2014; Righter et al. 2015). In Itokawa particles, olivine was found with an average compositional range of $\mathrm{Fa} 28.6$, low-Ca pyroxene of $\mathrm{Fs} 23.1$, and high- Ca pyroxene of Fs 8.9 (Nakamura et al. 2011). The porosity values for Chelyabinsk range between 2 and $11 \%$, with an average value of $\sim 6 \%$ (Moyano-Cambero et al. 2017), which is higher than the Itokawa particles (see Table 3). The Itokawa and LL chondrite samples have porosities ranging from approximately 0 to $10 \%$ with an average of 1.5 and $1.9 \%$.

The values of hardness and elastic recovery $\left(U_{\mathrm{e}} / U_{\mathrm{tot}}\right)$ fall in the same range as those measured for the Chelyabinsk meteorite. This can be understood bearing in mind that these two parameters are less sensitive to porosity than the Young's modulus.

In view of the mechanical properties of Itokawa's regolith particles we expect that these data can be used in future modeling of impact plume formation, and also to interpret the outcome of previous experiments (Michikami et al. 2007; Flynn et al. 2015). If micron-sized particles are strong enough and bear similar mechanical properties to those of meteorites, we expect that excavating a crater using a kinetic impactor in an area with significant fine-grained regolith will increase the momentum transfer. The reason is that the amount of micron-sized dust released in the creation of the impact plume will naturally increase the total target mass released without the need of grinding up the target during the mechanical excavation phase. Additional experiments to infer the velocity distribution of finegrained regolith particles, from a few to a hundred micron in size should be done with chondritic targets as was done previously (Nakamura et al. 1994; Flynn et al. 2015). The Double Asteroid Redirection Test (DART) mission plans to excavate a crater in the surface of the (65803) Didymos satellite; our results suggest that this excavation with a kinetic impactor in a significant finegrained regolith area will increase the momentum transfer and release particles in the direction opposite to the movement of the projectile. This would happen without the need of grinding up the target during the mechanical excavation.

\section{Conclusions}

The mechanical properties of micron-sized regolith particles of the asteroid Itokawa were studied by using the nanoindentation technique. Given that Hayabusa returned samples come from a rubble-pile near-Earth asteroid (NEA), it should be noted that the inferred physical properties of these particles are not very different to those exhibited by meteorites with similar ordinary chondrite composition. Our study provides a method that provides an accurate measurement of the mechanical response of regolith grains on the surface of chondritic asteroids. This is relevant because fine-grained regolith similar to this can be covering 
the surface of the satellite of asteroid 65803 Didymos planned to be impacted by the Double Asteroid Redirection Test (DART) NASA Mission in 2022. The deflection efficiency depends on our ability to release materials in the impact plume in the direction opposite to that of the projectile movement (see MoyanoCambero et al. 2017), so knowing the nature and mechanical properties of the rock-forming materials is of great importance. The conclusions of our current work are summarized as follows:

1. The nanoindentation technique can be successfully applied to study micron-sized particles returned in sample-return missions, providing very valuable information about the physical properties of the materials forming asteroids.

2. We find that the mechanical properties of Itokawa regolith particles are comparable with the silicates forming LL chondrite meteorites, with minor differences.

a. The reduced Young's modulus values obtained for the Itokawas samples are higher than those measured for Chelyabinsk chondrite, so they seem to be more compacted than the minerals forming that particular LL chondrite. This might be a natural consequence of the particles having survived long exposure times on the surface of a NEA.

b. The elastic recovery of Chelyabinsk chondrite minerals is significantly lower than that measured for the Itokawa samples, whereas the hardness values are similar. This indicates that the Itokawa particles have a greater ability to absorb elastic energy during an eventual impact than the Chelyabinsk chondrite minerals.

3. The inferred properties of micron-sized Hayabusa particles offer insights into the cohesion and mechanical response of regolith material on an asteroid surface. This is particularly relevant in view of the recent successful touchdown and sampling, and of the projectile impact of Hayabusa2 on asteroid 162173 Ryugu used to quantify the efficiency of crater excavation. It is important to note that the momentum transfer by a projectile on an asteroid depends on the mechanical properties of the surface materials: the amount of mass in the impact plume, released in the opposite direction to the projectile movement, is directly proportional to the deflection achieved.

4. Our results suggest that there is one important reason why excavating a crater via a kinetic impactor in an area with significant fine-grained regolith will increase the momentum transfer: the amount of micron-sized dust released in the creation of the impact plume, as a consequence of the mechanical excavation, will naturally increase the total target mass released even without grinding up the target.

A direct application of the parameters measured in this study will be the characterization of the impact outcome of the NASA DART spacecraft with the moon of the asteroid binary Didymos in October 2022. DART will perform the first deflection experiment on an asteroid. We will then be able to test our modeling of crater excavation and plume formation with a real deflection experiment. Obviously, the measurements of the dimensions of the crater will be required and this can only be achieved by another spacecraft examining the impact outcome after the DART mission. This is the objective of the ESA Hera mission; a candidate ESA mission that will be humankind's first probe to rendezvous with a binary asteroid system (Michel et al. 2018).

Acknowledgements. This study was supported by the Spanish grants AYA 201567175-P and AYA PGC2018-097374-B-I00 (PI: J.M.T-R.), and S.T. made this study in the frame of a Ph.D. on Physics at the Autonomous University of Barcelona (UAB). S.T. acknowledges travel support from JAXA and IEEC. P.M. acknowledges support from the French space agency CNES. Partial financial support from the 2017- SGR-292 project from the Generalitat de Catalunya and the SPIN-PORICS 2014-Consolidator Grant (Agreement No. 648454) from the European Research Council is acknowledged. We also thank Dr. Toru Yada and the Hayabusa staff of JAXA for providing the samples analyzed here.

\section{References}

Abe, M., Takagi, Y., Kitazato, K., et al. 2006, Science, 312, 1334

Beitz, E., Blum, J., Parisi, M. G., \& Trigo-Rodriguez, J. 2016, ApJ, 824, 12 Burbine, T. 2002, Bull. Czech Geolog. Survey, 77, 243

Chapman, C. R. 2002, In Asteroids III (Tucson: University of Arizona Press), 315

Chapman, C. R. 2004, Annu. Rev. Earth Planet. Sci., 32, 539

Delbo, M., Libourel, G., Wilkerson, J., et al. 2014, Nature, 508, 233

Fischer-Cripps, A. C. 2004, Nanoindentation (Switzerland: Springer Nature), 147 Flynn, G. J., Durda, D. D., Patmore, E. B., et al. 2015, Planet. Space Sci., 107, 64 Galimov, E., Kolotov, V., Nazarov, M., et al. 2013, Geochem. Int., 51, 522 Hiroi, T., Pieters, C. M., \& Takeda, H. 1994, Meteoritics, 29, 394

Kohout, T., Gritsevich, M., Grokhovsky, V. I., et al. 2014, Icarus, 228, 78

Matsumoto, T., Tsuchiyama, A., Uesugi, K., et al. 2016, Geochim. Cosmochim. Acta, 187, 195

Michel, P., Kueppers, M., Sierks, H., et al. 2018, Adv. Space Res., 62, 2261

Michikami, T., Moriguchi, K., Hasegawa, S., \& Fujiwara, A. 2007, Planet. Space Sci., 55,70

Moyano-Cambero, C. E., Pellicer, E., Trigo-Rodríguez, J. M., et al. 2017, ApJ, 835,157

Nakamura, A. M., Fujiwara, A., \& Kadono, T. 1994, Planet. Space Sci., 42, 1043

Nakamura, T., Noguchi, T., Tanaka, M., et al. 2011, Science, 333, 1113

Nix, W. D., \& Gao, H. 1998, J. Mech. Phys. Solids, 46, 411

Noguchi, T., Nakamura, T., Kimura, M., et al. 2011, Science, 333, 1121

Oliver, W. C., \& Pharr, G. M. 1992, J. Mater. Res., 7, 1564

Papike, J., Simon, S. B., \& Laul, J. 1982, Rev. Geophys., 20, 761

Pellicer, E., Pané, S., Panagiotopoulou, V., et al. 2012, Int. J. Electrochem. Sci., 7, 4014

Ramakrishnan, N., \& Arunachalam, V. 1993, J. Am. Ceram. Soc., 76, 2745

Righter, K., Abell, P., Agresti, D., et al. 2015, Meteorit. Planet. Sci., 50, 1790

Saito, J., Miyamoto, H., Nakamura, R., et al. 2006, Science, 312, 1341

Tolu, E., Garroni, S., Pellicer, E., et al. 2013, J. Mater. Chem. C, 1, 4948

Tsuchiyama, A., Uesugi, M., Uesugi, K., et al. 2014, Meteorit. Planet Sci., 49, 172

Yano, H., Kubota, T., Miyamoto, H., et al. 2006, Science, 312, 1350

Yomogida, K., \& Matsui, T. 1983, J. Geophys. Res. Solid Earth, 88, 9513 